\title{
Speech Enhancement in Hearing Aids Using Conjugate Symmetry of DFT and SNR-Perception Models
}

\author{
Dr. (Smt). S.D. Apte \\ Department of \\ Electronics and Communication Engineering \\ Rajarshi Shahu College of Engineering. \\ Pune, India.
}

\author{
Shridhar \\ Department of \\ Electronics and Communication Engineering \\ Basaveshwar Engineering College. \\ Bagalkot, India.
}

\begin{abstract}
Most of the speech enhancement algorithms use the magnitude of STFT while phase is kept unchanged [3]. In this paper the magnitude of STFT of noisy speech is kept unchanged while the phase is modified. Modified complex spectrum of speech is obtained by combining unchanged magnitude spectrum and modified phase spectrum [3]. This modification results into cancellation of low energy components (noise) of complex spectrum more than the high-energy (speech) components leading to reduction in background noise which is essential in hearing aids. SNR-Perception models for AWGN, Train noise, and Babble noise are developed using Nonlinear Regression modeling technique. Normal hearing and hearing loss subjective listening tests and spectrogram analysis show that the proposed method gives improved speech quality.
\end{abstract}

\section{Categories and Subject Descriptors}

[Speech/Audio]: Noise reduction, DFT, Conjugate symmetry, SNR-Perception models, Speech enhancement.

\section{General Terms}

Algorithms, Design, Performance, Experimentation, Human Factors, Theory, Verification.

\section{Keywords}

Hearing Aids, Speech enhancement, DFT, magnitude spectrum, phase spectrum, Conjugate symmetry.

\section{INTRODUCTION}

Speech intelligibility and quality, which are very important for hearing loss people, can be improved by its enhancement $[10,11,12,13]$. Hearing Aids supported by speech enhancement algorithms really help the hearing loss people to understand the speech in various noisy environments. In this direction, lot of research has been carried out [5, 2, 4]. From literature survey we find following speech enhancement methods.

1. Spectral subtraction. 2. Modified Spectral Subtraction.

3. Mean Square Estimation.4. Formant based methods.

5. Signal subspace Approach. 6. Wiener filtering

7. Modified Spectral Subtraction with masking property.

8. Kalman filtering. 9. Warped DFT based methods.

10. Human auditory properties (Masking) based methods

Let us consider a noisy speech signal

$$
\mathrm{x}(\mathrm{n})=\mathrm{s}(\mathrm{n})+\mathrm{N}(\mathrm{n})
$$

Assuming the noise to be additive. Where $x(n)=$ Noisy speech signal, $N(n)=$ noise. Frame wise analysis of the speech signal is carried out on the assumption that it is quasi stationary $[3,6,8,9]$. This assumption leads to the use of STFT for the signal processing. Applying STFT to the noisy speech signal x (n) we get

$$
X(k)=\sum_{m=-\infty}^{\infty} x(m) w(n-m) e^{-i 2 \pi k m / N}
$$

Here $n, k, w(n)$ are frame duration, discrete frequency index and window function respectively. Hamming window of $20 \mathrm{~ms}$ duration is used. Equation (1.1) can be written as $\mathrm{X}(\mathrm{k})=\mathrm{S}(\mathrm{k})+\mathrm{N}$ (k) where $\mathrm{X}(\mathrm{k}), \mathrm{S}(\mathrm{k})$ and $\mathrm{N}(\mathrm{k})$ are STFT of noisy speech, clean speech and noise respectively [1]. In the conventional speech enhancement methods the magnitude spectrum of noisy speech STFT is processed whereas phase spectrum is kept as it is. The processed magnitude spectrum is combined with unprocessed phase spectrum during signal synthesis. In the proposed work, the magnitude of noisy speech STFT is not processed, whereas its phase is modified based on empirical criteria [3]. The unprocessed magnitude is combined with modified phase during synthesis. This results into cancellation of low energy (noise) components more than high energy (speech) components $[3,14,15]$. This leads to enhancement of speech and is comparable with existing algorithms used in hearing aids. It is verified by subjective listening tests and spectrogram analysis. The paper is organized as follows. Section: 2 gives details of the proposed work. Section: 3 deals with modeling of SNR-Perception. Section: 4 deals with experimental details. Section: 5 presents results and discussion and finally section: 6 present the conclusions and future scope of the work.

\section{PROPOSED METHOD}

\subsection{Principle}

The noisy speech signal $\mathrm{x}$ (n) is real; hence its DFT obeys conjugate symmetry property. The same noisy speech signal $\mathrm{x}$ (n) is obtained if IDFT is computed straightaway due to cancellation of imaginary parts of complex conjugate terms of DFT, but the degree of cancellation or reinforcement of these imaginary parts of complex conjugates is controlled by modifying their phase. 


\subsection{Explanation}

The proposed method includes three stages of processing i.e. 1. Analysis stage. 2. Spectrum modification stage. 3. Synthesis stage [3]. The algorithm of the proposed method is given below:

Step1. Framing speech samples using Hamming Window with frame size of $20 \mathrm{msec}$ and $50 \%$ overlap

Step2. Computation of 1024 point DFT of each frame

Step3. Magnitude computation of each DFT bin.

Step4. Phase modification empirically.

Step5.Computation of modified spectrum using magnitude of Step 3 and phase of step 4.

Step 6.Computation of IDFT of each DFT bin.

Step7. Synthesis using overlap-add method.

The input noisy speech signal $\mathrm{x}(\mathrm{n})$ is real, hence its DFT obeys conjugate symmetry i.e. $X(k)=X^{*}(N-k)$. Straightaway IDFT of $\mathrm{X}(\mathrm{k})$ results into original noisy speech signal $\mathrm{x}(\mathrm{n})$ due to cancellation of imaginary parts of complex conjugate terms. But the degree of cancellation or reinforcement of complex conjugates can be controlled by modifying their phase [3]. A constant $\alpha(\mathrm{k})$, which is given by

$$
\begin{gathered}
\alpha(k)=\mathrm{c} ; 0 \leq k<N / 2 \\
\alpha(k)=-\mathrm{c} ; N / 2 \leq k \leq N-1 .
\end{gathered}
$$

Assuming $\mathrm{N}$ to be even, $\alpha(\mathrm{k})$ is a real valued frequency dependent function which is anti symmetric about the frequency Fs/2 rad/sample and $\mathrm{c}$ is a real valued constant [3]. The noisy speech signal STFT $\mathrm{X}(\mathrm{k})$ is modified as

$$
X \alpha(k)=X(k)+\alpha(k)
$$

The modified phase of $\mathrm{X} \alpha(\mathrm{k})$ is computed and further combined with magnitude of original noisy speech signal to get modified complex spectrum given by

$$
X m(k)=|X(k)| e^{i \angle X \alpha(k)}
$$

The IDFT of above complex spectrum results into enhanced real signal, the explanation is as follows. Fourier analysis resolves a signal $\mathrm{s}(\mathrm{t})$ into a weighted sum of sinusoidals that is to say a sum of complex conjugates. The magnitude spectrum of DFT of a real valued signal obeys even symmetry whereas phase spectrum obeys odd symmetry. During the process of signal synthesis (IDFT) the conjugates sum together to result into a real signal due to cancellation of their imaginary parts. The degree of cancellation or summation of these complex conjugates can be controlled by modifying their phase to address a particular hearing loss problem. The above process can be visualized using signal vector analogy. Considering a pair of complex conjugate numbers $\mathrm{C} 1=\mathrm{X}+\mathrm{j} \mathrm{Y}$ and $\mathrm{Cl}^{*}=\mathrm{X}-\mathrm{j} \mathrm{Y}$ having same magnitude

$$
M I=\sqrt{X^{2}+Y^{2}}
$$

and phase angles

$$
\begin{gathered}
\phi 1=\tan ^{-1}(Y / X) \\
\text { and } \phi 1^{*}=\tan ^{-1}(-Y / X)
\end{gathered}
$$

These complex conjugate numbers are modified as

$$
\mathrm{C} 11=X+j Y+C
$$

and $\quad C 11^{*}=X-j Y-C$

The resulting phase angles are

$$
\left.\phi 11=\tan ^{-1}(Y / X+C)\right)
$$

and $\quad \phi 11^{*}=\tan ^{-1}(-Y / X-C)$.

Combining the magnitude in equation (2.5) with phase in equations (2.10) and (2.11) forms the new complex conjugate numbers which can be expressed in polar form as

$$
\begin{aligned}
\mathrm{C} 1 \mathrm{P} & =\sqrt{X^{2}+Y^{2}} e^{i \tan ^{-1}(Y / X+C)} \\
\text { and } & \mathrm{C}^{*} \mathrm{P}^{*}=\sqrt{X^{2}+Y^{2}} e^{i \tan ^{-1}(-Y / X-C)}
\end{aligned}
$$

The resultant of above two complex conjugate numbers is given by

$$
C_{R I}=2 \sqrt{X^{2}+Y^{2}} ; \quad \text { If } \mathrm{C}<<M 1 .
$$

The resultant obtained in equation (2.14) is same as the resultant of original complex numbers, which is shown below. Original complex numbers are

$$
\begin{gathered}
C 1=X+j Y=\sqrt{X^{2}+Y^{2}} e^{i \tan ^{-1}(Y / X)} \\
C 1^{*}=X-j Y=\sqrt{X^{2}+Y^{2}} e^{i \tan ^{-1}(-Y / X)}
\end{gathered}
$$

The Resultant of above two complex numbers is given by

$$
C=2 \sqrt{X^{2}+Y^{2}}
$$

Therefore from equation (2.14) and equation (2.17) it is proved that $\mathrm{C}_{\mathrm{R} 1}=\mathrm{C}$. This implies that the phase modification due to $\mathrm{C}$ has very negligible effect on the spectral components having magnitude more than magnitude of $\mathrm{C}$. That is to say spectral components having magnitude more, nothing but speech components remain unaltered after phase modification as described above. The result is different when $\mathrm{C}>>\mathrm{M} 1$. The explanation is as follows. On the same lines considering another pair of complex conjugate numbers

$$
\begin{aligned}
& C 2=P+j Q \\
& C 2^{*}=P-j Q
\end{aligned}
$$

Both having the same magnitude given by

$$
M 2=\sqrt{P^{2}+Q^{2}}
$$

and phase angles

$$
\phi 2=\tan ^{-1}(Q / P)
$$

and

$$
\phi 2^{*}=\tan ^{-1}(-Q / P)
$$

respectively. These complex conjugate numbers are modified as

$$
\begin{aligned}
C 22 & =P+j Q+C \\
\text { and } \quad C 22^{*} & =P-j Q-C .
\end{aligned}
$$

The resulting phase angles are

$$
\phi 22=\tan ^{-1}(Q / P+C)
$$

and $\quad \phi 22^{*}=\tan ^{-1}(-Q / P-C)$

Combining the magnitude in equation (2.20) with phase in equations (2.25) and (2.26), forms the new complex conjugate numbers which can be expressed in polar form as

$$
C 2 P=\sqrt{P^{2}+Q^{2}} e^{i \tan ^{-1}(Q / P+C)}
$$


And $\quad C 2 P^{*}=\sqrt{P^{2}+Q^{2}} e^{i \tan ^{-1}(-Q / P-C)}$

The resultant of above two complex conjugate numbers is given by

$$
C_{R 2}=\sqrt{P^{2}+Q^{2}+P^{2}+Q^{2}+2\left(P^{2}+Q^{2}\right) \cos (\theta)}
$$

Where $\theta=\tan ^{-1}(Q / P+C)+\tan ^{-1}(-Q / P-C)$

If $\mathrm{C}>\mathrm{M} 2$, above equation (2.29) becomes

$$
\begin{aligned}
& \left.\left.C_{R 2}=\sqrt{2\left(P^{2}+Q^{2}\right)\left[1+\cos \left(\tan ^{-1}\right.\right.}\left[\frac{2 Q C}{C^{2}-Q^{2}}\right]\right)\right] \\
& C_{R 2<<} \sqrt{P^{2}+Q^{2}}
\end{aligned}
$$

Therefore from equations (2.20) and (2.32) it is proved that $\mathrm{C}_{\mathrm{R} 2}$ $<<$ M2. This implies that the phase modification due to $\mathrm{C}$ has considerable effect on the spectral components having magnitude less than magnitude of $\mathrm{C}$. That is to say spectral components having magnitude less (noise components) gets suppressed more after phase modification leading to enhancement of speech. This is based on the assumption that magnitudes of speech components are more than noise components in given noisy speech signal. The final outcome is, a particular value of $\mathrm{C}$ induces a definite value of phase modification in conjugate symmetrical spectral components leading to suppression of small spectral components (noise) and keeping the larger spectral components (speech) unaltered. This entire process leads to enhancement of speech, which is more beneficial to hearing loss people through their hearing aids. The various values of $\mathrm{C}$ for different types of noise are obtained empirically [3] and is given in table 3 .

\section{SNR-PERCEPTION MODELING.}

The value of $\mathrm{C}$ for a given value of SNR is obtained empirically. In this work three Nonlinear Regression models for $\mathrm{C}$ as a function of 1.Additive White Gaussian Noise 2. Train noise 3. Babble noise are obtained. The Models track the empirical values of $\mathrm{C}$ with negligible error. The model details are given below. The conventions used are $\mathrm{y}=\mathrm{C}, \mathrm{x}=\mathrm{SNR}(\mathrm{dB})$.

The model equation in case of AWGN is given by

$\mathrm{y}=1.82 * 10^{\wedge}(-6)^{*} \mathrm{x}^{\wedge} 5-1.49 * 10^{\wedge}(-4) * \mathrm{x}^{\wedge} 4+3.22 * 10^{\wedge}(-3) * \mathrm{x}^{\wedge} 3-$

$$
1.51 * 10^{\wedge}(-2) * \mathrm{x}^{\wedge} 2-0.282 * \mathrm{x}+3.496
$$

with Minimum Error $=-0.0184$, Maximum Error $=0.0125$.

The model equation in case of Train Noise is given by $\mathrm{y}=4.03 * 10^{\wedge}(-5)^{*} \mathrm{x}^{\wedge} 5-1.59^{*} 10^{\wedge}(-3)^{*} \mathrm{x}^{\wedge} 4+0.021 \mathrm{x}^{\wedge} 3-8.17^{*} 10^{\wedge}(-$

$$
\text { 2) } \mathrm{x}^{\wedge} 2-0.547 \mathrm{x}+5.99
$$

with Minimum Error $=-0.0078$, Maximum Error $=0.0108$.

The model equation in case of Babble Noise is given by $\mathrm{y}=3.59^{*} 10^{\wedge}(-5)^{*} \mathrm{x}^{\wedge} 5-1.54 * 10^{\wedge}(-3)^{*} \mathrm{x}^{\wedge} 4+2.13^{*} 10^{\wedge}(-2)^{*} \mathrm{x}^{\wedge} 3-$

$5.74 * 10^{\wedge}(-2) * \mathrm{x}^{\wedge} 2-1.137 * \mathrm{x}+9.531$

with Minimum Error $=-0.0386$, Maximum Error $=0.0342$.

The corresponding Model plots and Error plots are shown in figures 7-12.

\section{EXPERIMENTAL DETAILS 4.1. Speech database}

In the experimental evaluation the NOIZEUS speech corpus is used . This corpus is composed of non-stationary noises at different SNRs. In the evaluation, the train, babble and white Gaussian noises are used at $0 \mathrm{~dB}, 5 \mathrm{~dB}, 10 \mathrm{~dB}$ and $15 \mathrm{~dB}$ SNR levels.

\subsection{Experimentation Procedure}

Zero mean and normalized ( -1 to 1$)$ speech samples at a sampling frequency of $8 \mathrm{KHz}$ are obtained. 20ms frame duration and $10 \mathrm{~ms}$ overlap are chosen to frame the speech samples using Hamming window. 1024 point FFT of each frame is taken and its magnitude is computed. First $50 \%$ of the 1024 point FFT of each frame are modified by adding a constant $\mathrm{C}$ and remaining $50 \%$ of the samples are modified by subtracting the same constant $\mathrm{C}$. Here $\mathrm{C}$ is the function of speech SNR and type of noise. Phase of each modified DFT samples are calculated. Modified DFT values are obtained by combining the original DFT magnitudes and modified phase values. Finally IFFT is performed and discrete time signal is obtained using overlap add method [7]. The experiments were conducted using white noise, train noise and babble noise. Subjective test is carried on normal subjects and subjects with hearing loss for speech intelligibility assessment.

\section{RESSULTS AND DISCUSSION}

Mean opinion scores ( Table 1 and 2) obtained from listening tests on normal hearing subjects and subjects with hearing loss using speech samples S1, S2 and S3 show that the proposed method performs best in the case of white noise as compared to train and babble noise. The noisy speech sample is abbreviated as NS. The results of spectrogram analysis are shown in figures $1,2,3,4,5$ and 6. The enhanced speech (ES) signal in the presence of white Gaussian noise doesn't exhibit speech distortion. At the same time background noise is attenuated. In case of train and babble noise, though the background noise is suppressed, a small amount of speech distortion exists because of the factor $\mathrm{C}$ being constant for all values of frequencies within the frame. The SNR - Perception models for Gaussian, Train and Babble noise are developed using Nonlinear Regression Polynomial modeling. The model error is negligible in all the three cases.

\section{CONCLUSION AND FUTURE SCOPE OF WORK}

In this paper an improved method of speech enhancement is presented which finds a front-end application in hearing aids used by people suffering from general hearing loss due to age and sensori neural problems. The magnitude spectrum of noisy speech signal is combined with modified phase spectrum to get

modified complex spectrum. During signal synthesis using modified complex spectrum low energy components (noise) cancels out more as compared to high-energy components (speech). Thus achieving enhancement of speech. The work is validated through subjective listening tests on normal hearing subjects and subjects with hearing loss. The mean opinion score of normal hearing subjects and subjects with hearing loss differs by a small value. The reduction in noise is evident from the spectrograms of noisy speech and enhanced speech. As a future scope of work more experimental investigation can be done on 
subjects with hearing loss due to age, sensori neural hearing loss using SNR-Perception Models to understand human auditory system better.

Table1. Opinion Score and Mean Opinion Score of Speech Intelligibility Test on Normal Hearing Male (M) Subjects.

Table 2. Opinion Score and Mean Opinion Score of Speech Intelligibility Test on Hearing Loss (HL) Subjects.

\begin{tabular}{|c|c|c|c|c|c|c|c|c|c|c|c|c|c|c|c|c|c|c|c|c|c|c|}
\hline \multirow[b]{4}{*}{ NOISE } & \multirow[b]{4}{*}{$\begin{array}{l}\text { SNR } \\
(\mathrm{dB})\end{array}$} & \multicolumn{21}{|c|}{ SUBJECTIVE SPEECH INTELLIGIBILITY TEST } \\
\hline & & \multicolumn{6}{|c|}{$\begin{array}{c}\text { SUBJECT (M1) } \\
\text { OPINION SCORE }\end{array}$} & \multicolumn{6}{|c|}{$\begin{array}{c}\text { SUBJECT (M2) } \\
\text { OPINION SCORE }\end{array}$} & \multicolumn{6}{|c|}{$\begin{array}{c}\text { SUBJECT (M3) } \\
\text { OPINION SCORE }\end{array}$} & \multicolumn{3}{|c|}{$\begin{array}{l}\text { MEAN } \\
\text { OPINION } \\
\text { SCORE }\end{array}$} \\
\hline & & \multicolumn{2}{|c|}{ S1 } & \multicolumn{2}{|c|}{ S2 } & \multicolumn{2}{|c|}{ S3 } & \multicolumn{2}{|c|}{ S1 } & \multicolumn{2}{|c|}{ S2 } & \multicolumn{2}{|c|}{ S3 } & \multicolumn{2}{|c|}{ S1 } & \multicolumn{2}{|c|}{ S2 } & \multicolumn{2}{|c|}{ S3 } & \multirow{2}{*}{$\begin{array}{ll}\text { S1 } \\
\text { ES }\end{array}$} & \multirow{2}{*}{$\begin{array}{l}\text { S2 } \\
\text { ES }\end{array}$} & \multirow{2}{*}{$\begin{array}{l}\text { S3 } \\
\text { ES }\end{array}$} \\
\hline & & NS & ES & NS & ES & NS & ES & NS & ES & NS & ES & NS & ES & NS & ES & NS & ES & NS & ES & & & \\
\hline \multirow{4}{*}{ AWGN } & 00 & 1.0 & 1.5 & 1.0 & 1.5 & 1.0 & 1.5 & 1.0 & 2.0 & 1.0 & 2.0 & 1.0 & 2.0 & 1.0 & 1.5 & 1.0 & 1.6 & 1.0 & 1.5 & 1.6 & 1.6 & 1.6 \\
\hline & 05 & 2.0 & 3.0 & 2.0 & 2.2 & 2.0 & 2.6 & 1.5 & 3.0 & 2.0 & 3.0 & 2.0 & 3.0 & 2.0 & 3.0 & 2.0 & 2.5 & 2.8 & 3.0 & 3.0 & 2.5 & 2.8 \\
\hline & 10 & 3.0 & 4.0 & .0 & 3.5 & 3.0 & 3.8 & 3.0 & 4.0 & 3.0 & 4.0 & 3.0 & 4.0 & 3.0 & 4.0 & 3.0 & 4.0 & 3.0 & 4.0 & 4.0 & 3.8 & 3.9 \\
\hline & 15 & 3.5 & 4.5 & 3.8 & 4.3 & 3.7 & 4.4 & 3.5 & 4.5 & 4.0 & 4.5 & 4.0 & 5.0 & 3.5 & 4.5 & 4.0 & 4.5 & 4.0 & 4.5 & 4.5 & 4.4 & 4.4 \\
\hline \multirow{4}{*}{ TRAIN } & 00 & 1.0 & 2.5 & 1.0 & 3.2 & 1.0 & $2 . .9$ & 1.0 & 3.0 & 1.0 & 2.5 & 1.0 & 2.5 & 1.0 & 2.5 & 1.0 & 2.0 & 1.0 & 2.0 & 2.6 & 2.5 & 2.4 \\
\hline & 05 & 2.2 & 3.5 & 1.5 & 3.5 & 1.5 & 3.5 & 2.0 & 4.0 & 2.5 & 3.5 & 2.0 & 3.5 & 2.2 & 3.5 & 2.0 & 3.5 & 2.0 & 3.0 & 3.6 & 3.5 & 3.3 \\
\hline & 10 & 3.0 & 4.2 & 2.5 & 4.0 & 2.8 & 4.1 & 3.0 & 4.5 & 3.0 & 4.0 & 3.0 & 4.0 & 3.0 & 4.2 & 3.0 & 4.5 & 3.0 & 4.2 & 4.3 & 4.1 & 4.1 \\
\hline & 15 & 4.0 & 4.7 & 2.0 & 4.5 & 3.5 & 4.6 & 4.0 & 5.0 & 4.0 & 4.9 & 4.0 & 4.5 & 4.0 & 4.7 & 4.0 & 4.5 & 4.2 & 4.2 & 4.8 & 4.5 & 4.6 \\
\hline \multirow{4}{*}{ BABBLE } & 00 & 0.8 & 1.1 & 0.8 & 1.2 & 1.8 & 1.2 & 1.0 & 1.5 & 1.0 & 1.5 & 1.0 & 2.0 & 0.8 & 1.1 & 1.0 & 1.1 & 1.0 & 1.2 & 1.3 & 1.2 & 1.4 \\
\hline & 05 & 2.2 & 3.2 & 1.3 & 1.8 & 1.8 & 2.5 & 2.0 & 2.5 & 2.0 & 2.5 & 2.0 & 3.0 & 2.2 & 3.3 & 2.0 & 2.5 & 2.0 & 3.5 & 2.9 & 2.2 & 2.6 \\
\hline & 10 & 3.5 & 4.5 & 2.5 & 3.0 & 3.0 & 3.8 & 3.0 & 4.0 & 3.0 & 4.0 & 3.0 & 4.0 & 3.5 & 4.5 & 3.0 & 4.0 & 3.0 & 3.7 & 4.3 & 3.6 & 3.8 \\
\hline & 15 & 4.0 & 4.8 & 3.8 & 4.2 & 3.9 & 4.5 & 4.0 & 4.5 & 4.0 & 4.5 & 4.0 & 4.9 & 4.0 & 4.8 & 4.0 & 4.5 & 4.0 & 4.9 & 4.7 & 4.4 & 4.7 \\
\hline
\end{tabular}

\begin{tabular}{|c|c|c|c|c|c|c|c|c|c|c|c|c|c|c|c|c|c|c|c|c|c|c|}
\hline \multirow[b]{4}{*}{ NOISE } & \multirow[b]{4}{*}{$\begin{array}{l}\text { SNR } \\
\text { (dB) }\end{array}$} & \multicolumn{21}{|c|}{ SUBJECTIVE SPEECH INTELLIGIBILITY TEST } \\
\hline & & \multicolumn{6}{|c|}{$\begin{array}{l}\text { SUBJECT (HL1) } \\
\text { OPINION SCORE }\end{array}$} & \multicolumn{6}{|c|}{$\begin{array}{l}\text { SUBJECT (HL2) } \\
\text { OPINION SCORE }\end{array}$} & \multicolumn{6}{|c|}{$\begin{array}{l}\text { SUBJECT (HL3) } \\
\text { OPINION SCORE }\end{array}$} & \multicolumn{3}{|c|}{$\begin{array}{l}\text { MEAN } \\
\text { OPINION } \\
\text { SCORE }\end{array}$} \\
\hline & & \multicolumn{2}{|c|}{ S1 } & \multicolumn{2}{|c|}{ S2 } & \multicolumn{2}{|c|}{ S3 } & \multicolumn{2}{|c|}{ S1 } & \multicolumn{2}{|c|}{ S2 } & \multicolumn{2}{|c|}{ S3 } & \multicolumn{2}{|c|}{ S1 } & \multicolumn{2}{|c|}{ S2 } & \multicolumn{2}{|c|}{ S3 } & \multirow{2}{*}{$\begin{array}{l}\text { S1 } \\
\text { ES }\end{array}$} & \multirow{2}{*}{$\begin{array}{l}\text { S2 } \\
\text { ES }\end{array}$} & \multirow{2}{*}{ S3 } \\
\hline & & NS & ES & NS & ES & NS & ES & NS & ES & NS & ES & NS & ES & NS & ES & NS & ES & NS & ES & & & \\
\hline \multirow{4}{*}{ AWGN } & 00 & 2.0 & 1.0 & 2.0 & 1.0 & 1.0 & 2.0 & 2.0 & 1.5 & 1.0 & 2.0 & 2.0 & 1.0 & 2.5 & 1.0 & 2.0 & 1.0 & 2.0 & 1.5 & 1.1 & 1.1 & 1.5 \\
\hline & 05 & 3.0 & 3.0 & 3.0 & 3.0 & 2.0 & 3.0 & 3.1 & 2.5 & 3.0 & 2.5 & 2.5 & 2.5 & 3.0 & 3.0 & 3.0 & 3.5 & 2.0 & 2.5 & 2.8 & 3.0 & 2.6 \\
\hline & 10 & 3.0 & 4.0 & 3.0 & 4.0 & 3.0 & 4.0 & 3.0 & 4.0 & 2.5 & 4.0 & 3.0 & 4.0 & 3.0 & 4.0 & 3.0 & 3.5 & 2.5 & 3.5 & 4.0 & 3.8 & 3.8 \\
\hline & 15 & 4.0 & 5.0 & 4.0 & 5.0 & 4.0 & 5.0 & 3.5 & 5.0 & 3.5 & 5.0 & 4.0 & 4.5 & 3.5 & 5.0 & 4.0 & 4.5 & 3.5 & 4.5 & 5.0 & 4.8 & 4.6 \\
\hline \multirow{4}{*}{ TRAIN } & 00 & 1.0 & 2.0 & 1.0 & 2.0 & 1.0 & 2.0 & 1.0 & 2.5 & 1.5 & 2.5 & 4.5 & 3.0 & 1.0 & 4.5 & 1.0 & 1.5 & 1.0 & 2.0 & 3.0 & 2.3 & 2.3 \\
\hline & 05 & 2.0 & 3.0 & 2.0 & 3.0 & 2.0 & 3.0 & 2.0 & 3.0 & 2.0 & 3.0 & 2.0 & 3.5 & 3.0 & 3.5 & 3.5 & 3.5 & 3.0 & 3.0 & 3.1 & 3.1 & 3.1 \\
\hline & 10 & 3.0 & 3.0 & 3.0 & 3.0 & 3.0 & 3.0 & 3.0 & 3.5 & 3.0 & 4.0 & 3.0 & 4.0 & 3.0 & 3.0 & 3.0 & 3.5 & 2.0 & 2.5 & 3.1 & 3.8 & 3.1 \\
\hline & 15 & 4.0 & 4.0 & 4.0 & 5.0 & 4.0 & 5.0 & 3.5 & 4.0 & 3.5 & 4.5 & 4.0 & 4.5 & 4.0 & 5.0 & 4.5 & 5.0 & 4.0 & 5.0 & 4.3 & 4.8 & 4.8 \\
\hline \multirow{4}{*}{ BABBLE } & 00 & 1.0 & 2.0 & 1.0 & 2.0 & 1.0 & 2.0 & 1.0 & 2.0 & 1.0 & 2.1 & 1.0 & 2.0 & 1.0 & 2.0 & 1.5 & 2.5 & 1.5 & 2.0 & 2.0 & 2.2 & 2.0 \\
\hline & 05 & 2.0 & 3.0 & 3.0 & 1.0 & 2.0 & 1.0 & 2.0 & 3.0 & 2.5 & 3.0 & 2.0 & 3.5 & 2.0 & 2.5 & 2.5 & 2.0 & 2.0 & 2.5 & 2.8 & 3.3 & 3.3 \\
\hline & 10 & 3.0 & $\begin{array}{l}4.0 \\
\end{array}$ & 2.0 & $\begin{array}{l}4.0 \\
\end{array}$ & $\begin{array}{l}3.0 \\
\end{array}$ & 4.0 & 2.5 & 3.5 & 2.5 & 3.5 & $\begin{array}{l}3.0 \\
\end{array}$ & $\begin{array}{l}4.0 \\
\end{array}$ & 2.5 & 3.5 & 3.0 & 3.5 & 3.0 & 3.5 & 3.5 & 3.6 & $\begin{array}{l}3.8 \\
\end{array}$ \\
\hline & 15 & 4.0 & 5.0 & 4.0 & 5.0 & 4.0 & 5.0 & 3.5 & 4.0 & 3.5 & 4.0 & 4.0 & 5.0 & 4.5 & 5.0 & 4.0 & 4.5 & 3.5 & 4.5 & 4.6 & 4.6 & 4.8 \\
\hline
\end{tabular}




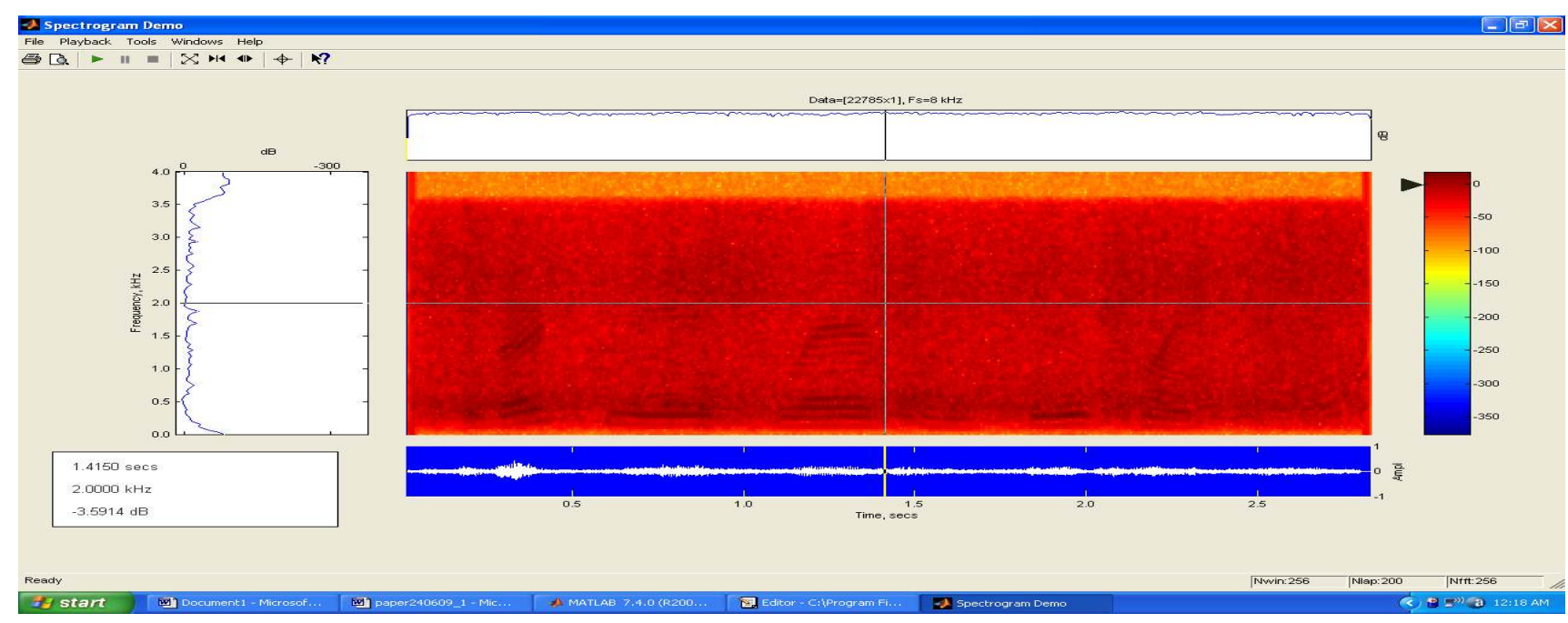

Figure 1. Spectrogram of noisy speech sample (Train noise) of SNR 0 dB from NOIZEUS data base.

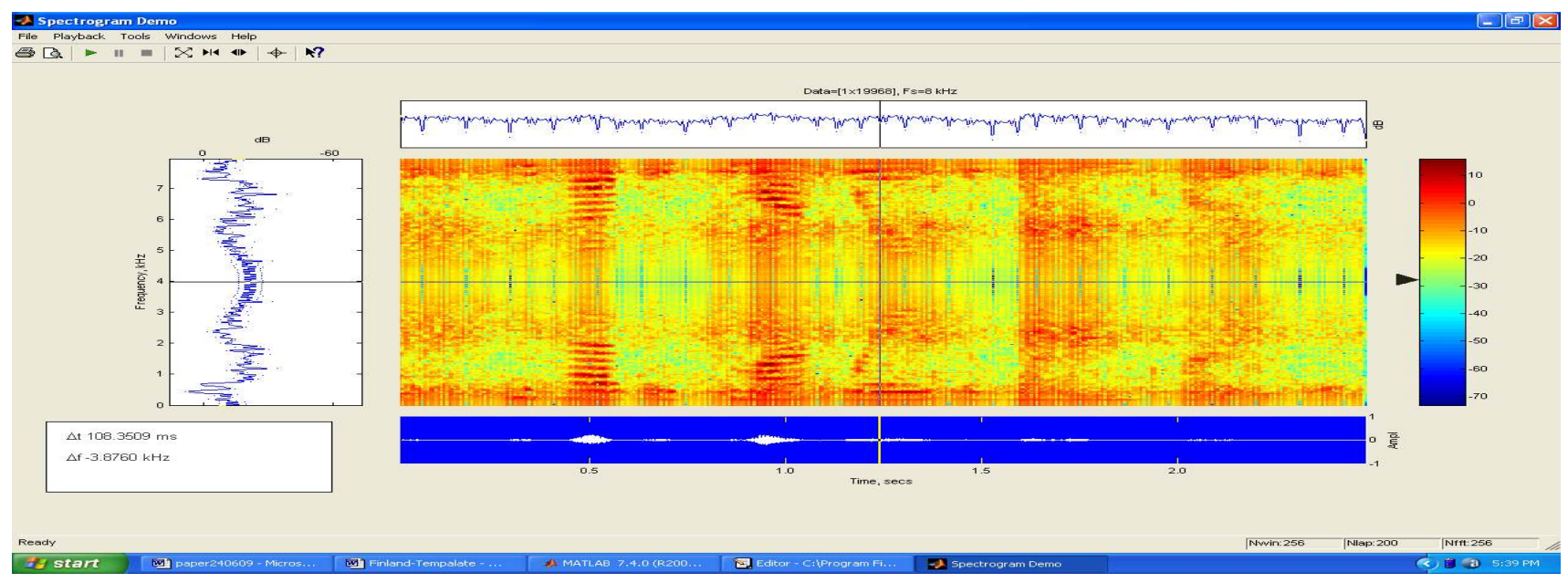

Figure 2. Spectrogram of enhanced speech sample of figure 1.

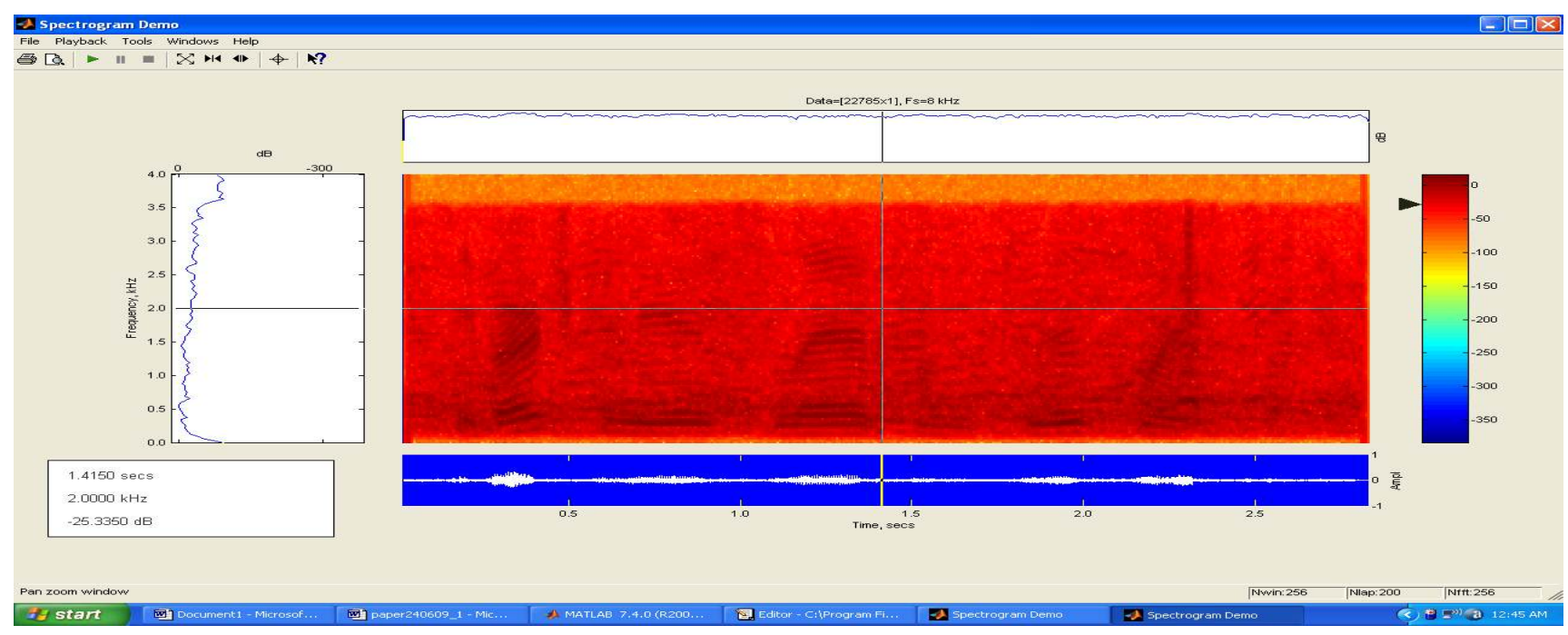


Figure 3. Spectrogram of noisy speech sample (Babble noise) of SNR $5 \mathrm{~dB}$ from NOIZEUS data base.

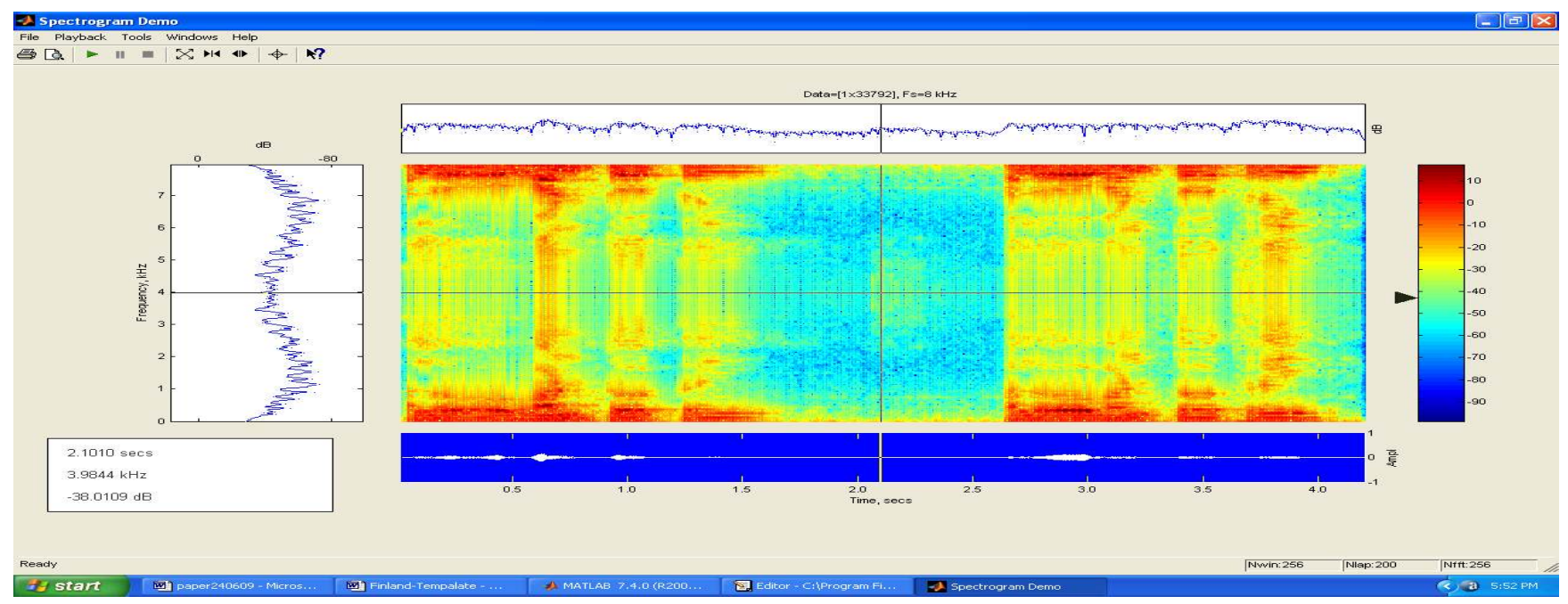

Figure 4. Spectrogram of enhanced speech sample of figure 3.

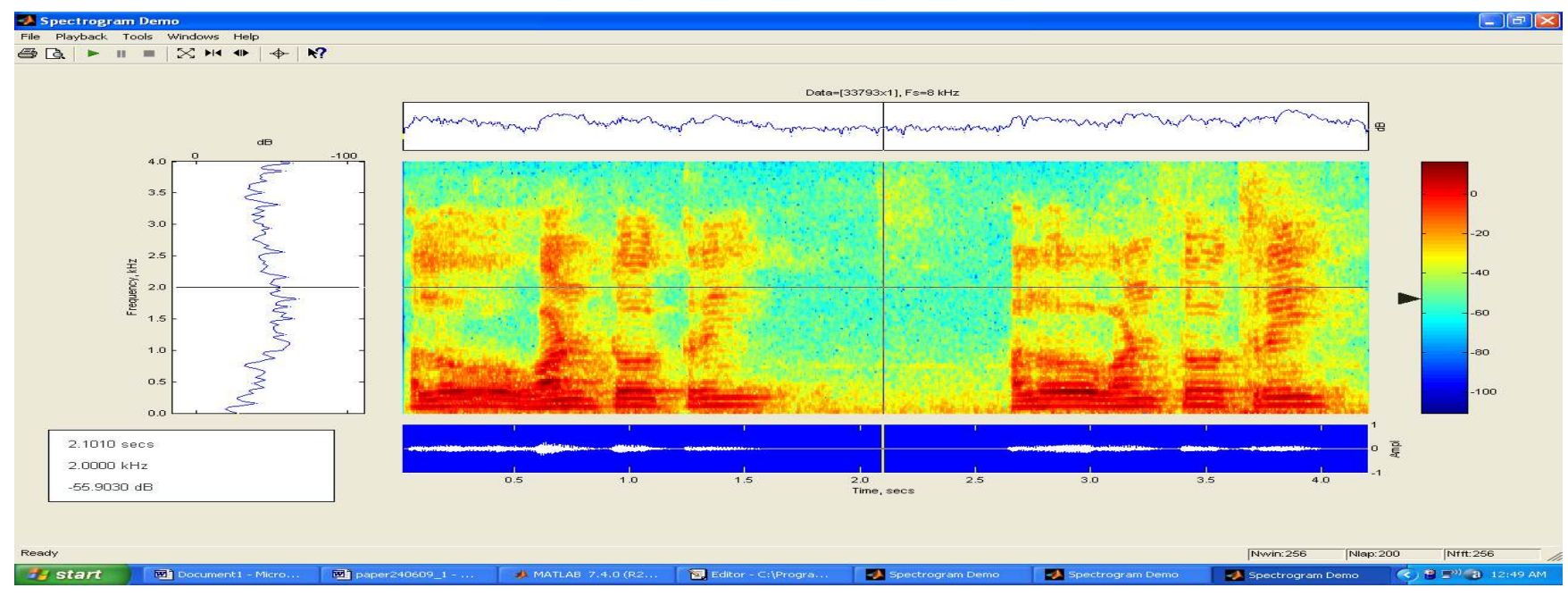

Figure 5. Spectrogram of noisy speech sample (Gaussian noise) of SNR $10 \mathrm{~dB}$ from NOIZEUS data base.

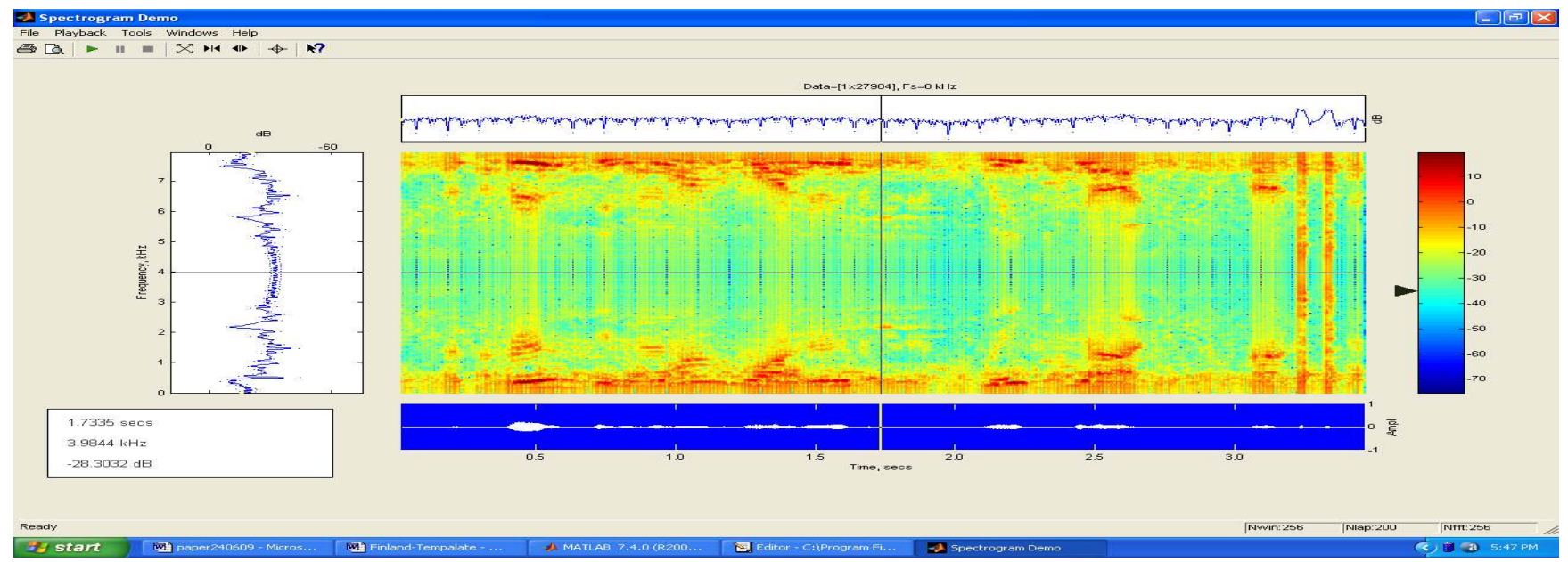


Figure 6. Spectrogram of enhanced speech sample of figure 5.

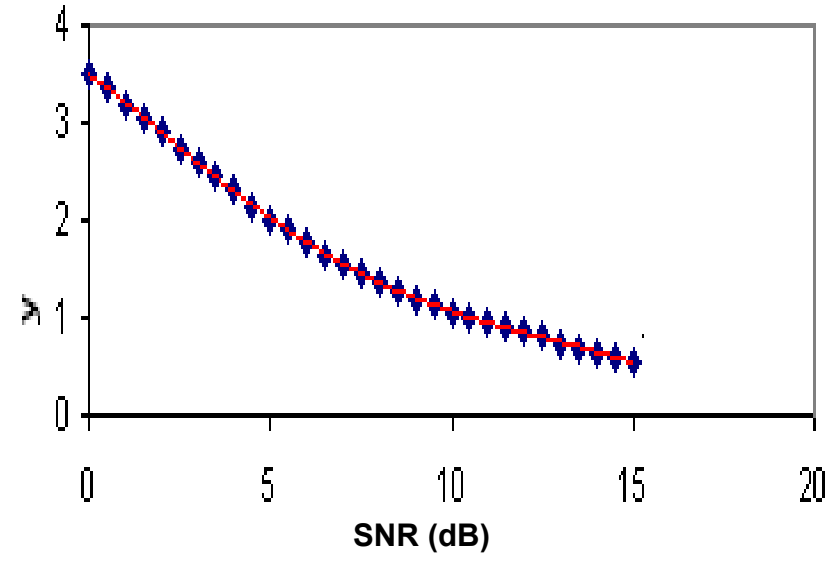

Figure 7. Empirical Model plot of C v/s Noise (AWGN). Model Equation $y=1.82^{\star} 10^{\wedge}(-6)^{\star} x^{\wedge} 5-1.49^{\star} 10^{\wedge}(-4)^{\star} x^{\wedge} 4+3.22^{\star} 10^{\wedge}(-3)^{\star} x^{\wedge} 3-1.51^{\star} 10^{\wedge}(-2)^{\star} x^{\wedge} 2-0.282^{\star} x+3.496$

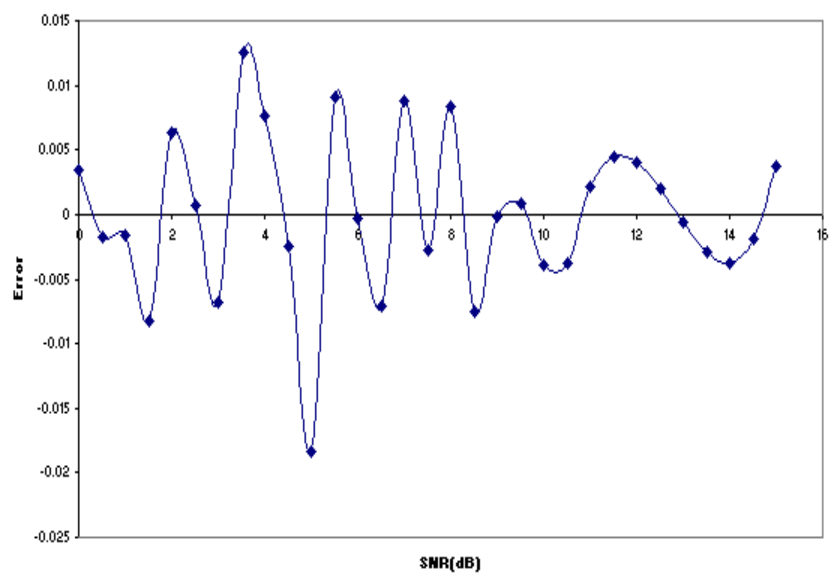

Figure8.Error Plot of Empirical Model for AWGN and C

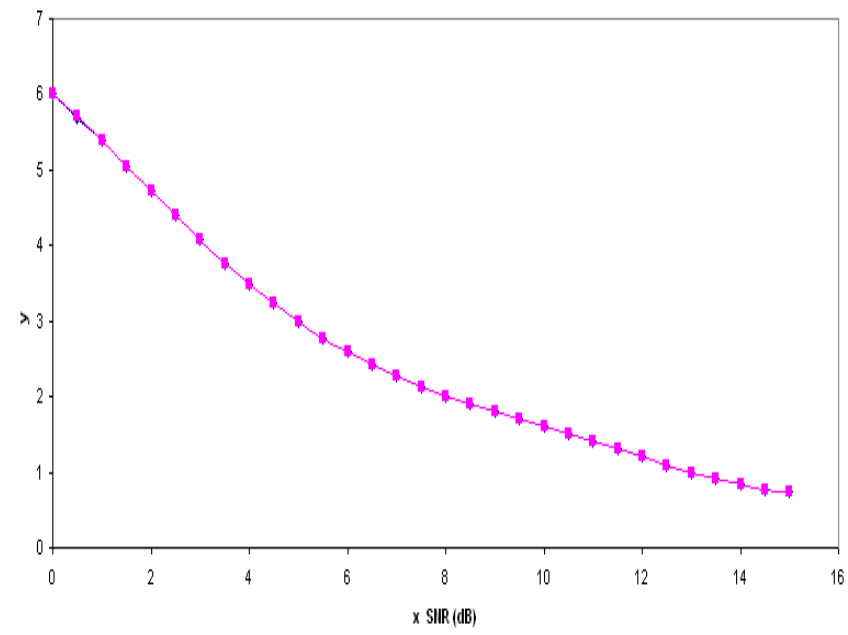

Figure 9. Empirical Model plot of C v/s Noise (AWGN). Model Equation $y=4.03^{\star} 10^{\wedge}(-5)^{\star} x^{\wedge} 5-1.59^{\star} 10^{\wedge}(-3)^{\star} x^{\wedge} 4+0.021 x^{\wedge} 3-8.17^{\star} 10^{\wedge}(-2) x^{\wedge} 2-0.547 x+5.99$

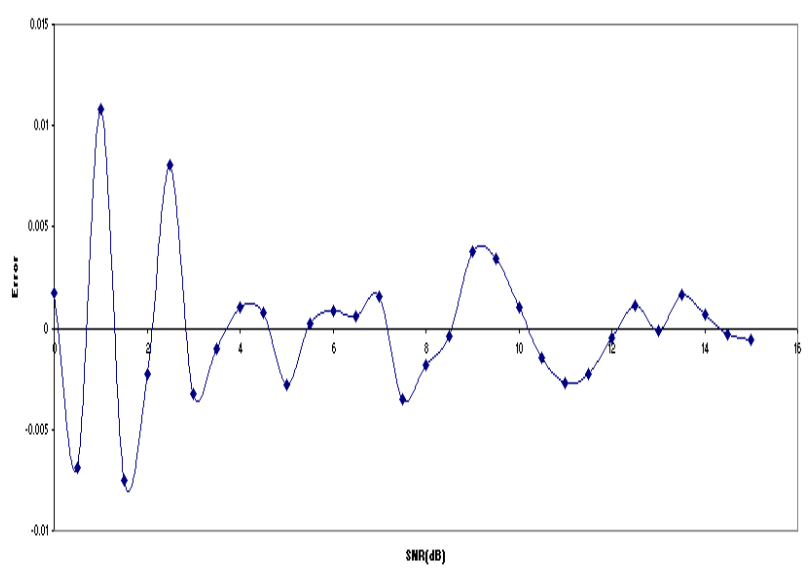

Figure10. Error Plot of Empirical Model for Train Noise and $\mathrm{C}$

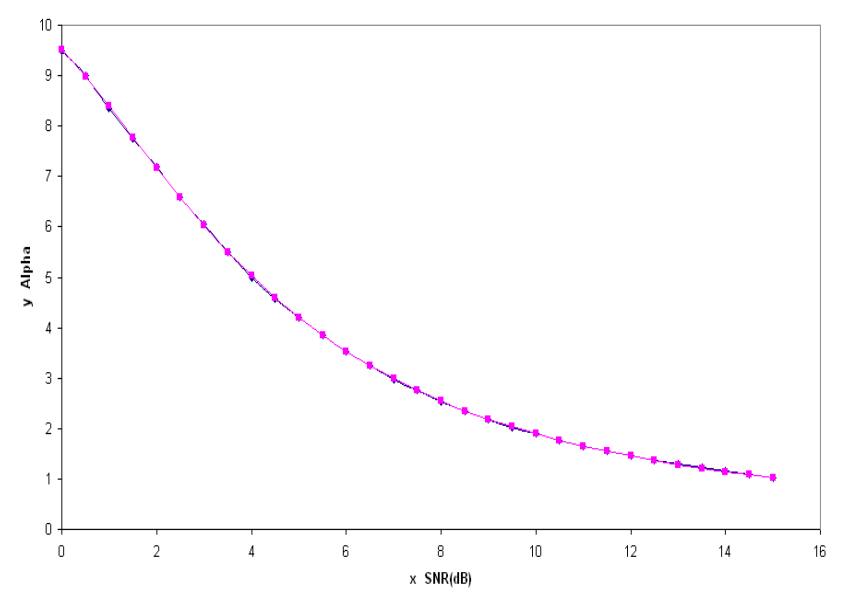

Figure 11. Empirical Model plot of $\mathrm{C}$ v/s Noise (Babble). Model Equation

$y=3.59^{*} 10^{\wedge}(-5)^{\star} x^{\wedge} 5-1.54^{*} 10^{\wedge}(-3)^{\star} x^{\wedge} 4+2.13^{*} 10^{\wedge}(-2)^{\star} x^{\wedge} 3.5 .74^{\star} 10^{\wedge}(-2)^{\star} x^{\wedge} 2-1.137^{\star} x+9.531$

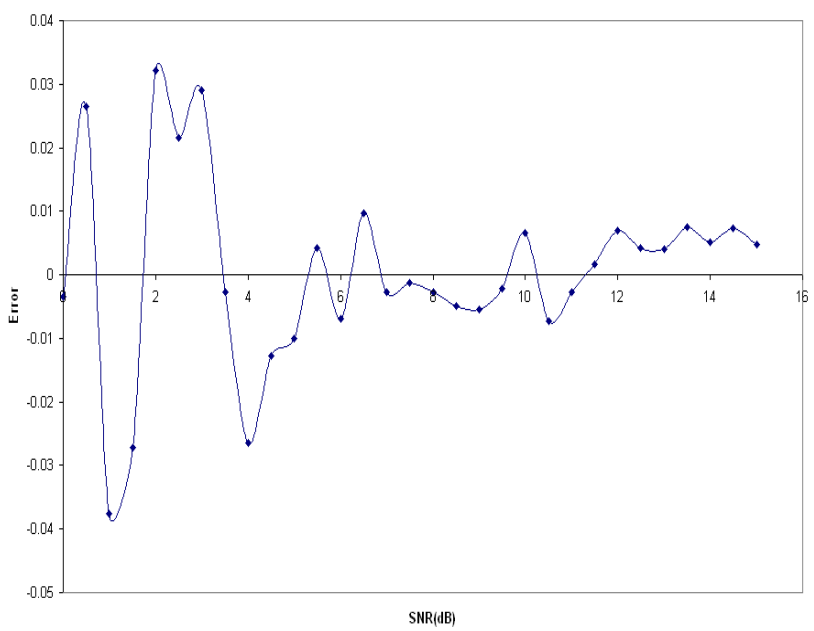

Figure 12. Error plot of Empirical Model for Babble noise and $\mathbf{C}$. 
Table 3: Empirical values of $\mathrm{C}$ for different Noise and SNR

\begin{tabular}{|c|c|c|c|}
\hline \multicolumn{4}{|c|}{ NOISE TYPE } \\
\hline SNR (dB) & AWGN & TRAIN & BABBLE \\
\hline 0.0 & 3.50 & 6.00 & 9.50 \\
\hline 0.5 & 3.35 & 5.70 & 9.00 \\
\hline 1.0 & 3.20 & 5.40 & 8.35 \\
\hline 1.5 & 3.00 & 5.00 & 7.75 \\
\hline 2.0 & 2.90 & 4.75 & 7.20 \\
\hline 2.5 & 2.75 & 4.40 & 6.60 \\
\hline 3.0 & 2.55 & 4.10 & 6.05 \\
\hline 3.5 & 2.45 & 3.80 & 5.50 \\
\hline 4.0 & 2.30 & 3.55 & 5.00 \\
\hline 4.5 & 2.15 & 3.20 & 4.40 \\
\hline 5.0 & 2.00 & 2.90 & 3.80 \\
\hline 5.5 & 1.97 & 2.75 & 3.60 \\
\hline 6.0 & 1.75 & 2.55 & 3.50 \\
\hline 6.5 & 1.65 & 2.45 & 3.25 \\
\hline 7.0 & 1.55 & 2.30 & 3.05 \\
\hline 7.5 & 1.50 & 2.15 & 2.80 \\
\hline 8.0 & 1.45 & 2.00 & 2.60 \\
\hline 8.5 & 1.35 & 1.85 & 2.50 \\
\hline 9.0 & 1.25 & 1.60 & 2.30 \\
\hline 9.5 & 1.15 & 1.50 & 2.05 \\
\hline 10.0 & 1.05 & 1.45 & 1.90 \\
\hline 10.5 & 1.00 & 1.35 & 1.80 \\
\hline 11.0 & 0.95 & 1.30 & 1.65 \\
\hline 11.5 & 0.90 & 1.25 & 1.55 \\
\hline 12.0 & 0.85 & 1.15 & 1.50 \\
\hline 12.5 & 0.80 & 1.05 & 0.40 \\
\hline 13.0 & 0.75 & 1.00 & 1.30 \\
\hline 13.5 & 0.70 & 0.95 & 1.20 \\
\hline 14.0 & 0.65 & 0.90 & 0.15 \\
\hline 14.5 & 0.60 & 0.85 & 0.95 \\
\hline 15.0 & 0.55 & 0.75 & 0.85 \\
\hline
\end{tabular}

\section{REFERENCES}

[1] S. Boll, "Suppression of acoustic noise in speech using spectral subtraction ,'IEEE Trans .Acoustics, speech, signal processing.,vol.ASSP-27,NO.2,PP.113-120, Apr.1979.

[2] Y.Ephraim and D. Malah, "Speech enhancement using a minimum mean -square error log-spectral amplitude estimator, "IEEE Trans. Acoustics, Speech Signal Processing, vol.ASSP-33, no.2, pp.443-445, Apr.1985.
Lyons, and Kuldip Paliwal, "Exploiting conjugate symmetry of the short - time Fourier spectrum for speech enhancement, "IEEE Signal processing letters, Vol.15.2008. pp.461-464

[4] K.Paliwal and A.Basu, "A Speech enhancement method based on kalman filtering", in proc. IEEE Int. Conf. Acoustics, Speech, and Signal Processing (ICASSP'87), Apr.1987, vol.12, pp.297 - 300.

[5] Y. Ephraim and H. V. Trees, "A Signal Subspace approach for speech enhancement" IEEE Trans. Speech Audio Processing.,vol.3,no.4. pp. 251-266, July.1995.

[6] J.Allen and L.Rabiner, "A Unified approach to shorttime Fourier analysis and synthesis", Proc. IEEE, vol. 65 , no.11, pp. 1558 -1564, Nov.1977.

[7] R.Crochiere, "A weighted overlap-add method of short -time Fourier analysis/ synthesis", IEEE Trans. Acoustics. Speech, Signal Processing., Vol ASSP-28,no.2, pp, 99- 102, Apr.1980

[8] D. Griffin and J. Lim, " Signal estimation from modified short-time Fourier Transform," IEEE Trans. Acoustics, speech, Signal processing. Vol. ASSP-32, no.2, pp.236 -243, Apr. 1984.

[9] M. Portnoff, "Short-time Fourier analysis of sampled speech, "IEEE Trans. Acoustics., Speech, Signal process., Vol. ASSP- 29, no.3, pp.364-373, Jun.1981.

[10] Richard J Baker, Stuart Rosen, “Auditory filter non linearity in mild/moderate hearing impairment. "Journal of Acoustical Society of America, 111(3), March 2002, pp.1330-1339.

[11] Pick G.F., Evans E.F. "Dissociation between frequency resolution and hearing threshold" In hearing-Physiological basis and Psychophysics, Edited by R. Klinke and R.Hartmann.pp.393-398.

[12] Rosen S. Baker R.J, Darling A.M. "Auditory filter non linearity at $2 \mathrm{kHz}$ in normal listeners" Journal of Acoustical Society of America, 103, pp.2539-2550

[13] Tyler R.S, Hall J.W, Glassberg B.R, Moore B.C.J, Patterson R.D. "Auditory filter asymmetry in the hearing impaired". Journal of Acoustical Society of America, 76, pp.1363-1368.

[14] B.C.J Moore. "Speech processing for the Hearing impaired: Successes, Failures and Implications for Speech mechanisms" Speech communication, Elsevier 41(2003) pp.81-91.

[15] Jesko L.Verhey, Anne Kristin Anweiler, Volker Hohmann, "Spectral loudness summation as a function of duration for hearing impaired listeners, International Journal of Audiology.2006; 45; pp.287-294.

[3] Kamil Wojcicki ,Mitar Milacic,Anthony Stark, James 